
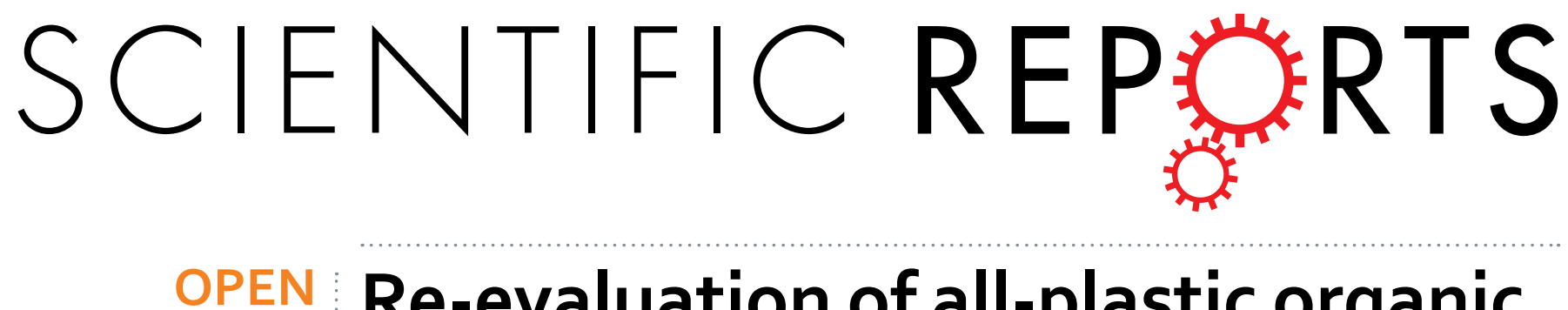

\title{
Re-evaluation of all-plastic organic dye laser with DFB structure fabricated using photoresists
}

Received: 11 July 2016

Accepted: 19 September 2016

Published: 05 October 2016

\section{Naoto Tsutsumi ${ }^{1}$, Saori Nagi ${ }^{2}$, Kenji Kinashi ${ }^{1}$ \& Wataru Sakai ${ }^{1}$}

Organic solid-state lasers (OSSLs) with distributed feedback structures can detect nanoscale materials and therefore offer an attractive sensing platform for biological and medical applications. Here we investigate the lasing characteristics, i.e., the threshold and slope efficiency, as a function of the grating depth in OSSL devices with distributed feedback (DFB) structure fabricated using photoresists. Two types of photoresists were used for the DFB structures: a negative photoresist, SU-8 2002, and a positive photoresist, ma-P 1275. The DFB structure was fabricated using a Lloyd-mirror configuration. The active layer was a rhodamine 6G-doped cellulose acetate waveguide. The threshold for the first order mode $(m=1)$ was lower than that for the second and third order modes $(m=2$, and 3$)$. A low threshold of $27 \mu \mathrm{J} \mathrm{cm} \mathrm{cm}^{-2}$ pulse ${ }^{-1}(58 \mathrm{~nJ})$ was obtained using SU-8 2002, with $m=1$. The slope efficiency was evaluated as a function of grating depth for each mode and increased as the grating depth increased.

In the past two decades, organic solid-state lasers (OSSLs) ${ }^{1-13}$ have been developed extensively because of the easy fabrication of laser devices by spin-coating or solution casting, the low cost of device fabrication, and the wide variation of the lasing wavelength, ranging from the visible to near infrared regions, that can be selected depending on the laser dye used. Slope efficiency is an important measure to evaluate the performance of the laser devices. The slope efficiency is commonly determined by the slope between the pump (input) energy and output energy. A high brightness OSSL with a slope efficiency of $11 \%$ was achieved using a 4-(dicyanomethylene)-2-met hyl-6-(4-dimethylaminostyryl)-4H-pyran (DCM)-based Vertical External-Cavity Surface-emitting Organic Laser (VECSOL) ${ }^{14}$. More recently, studies have shown that the organic thin film laser of the VECSOL can operate in single longitudinal mode (SLM) with the combination of a volume Bragg grating (VBG) ${ }^{15}$. Organic distributed feedback (DFB) dye lasers and distributed Bragg reflector (DBR) dye lasers also have specific features of SLM emissions. An OSSL possessing degradation recovery and based on polydimethylsiloxane as the host matrix was reported. Its durability increased 20.5-fold compared with that of an OSSL based on poly (methyl methacrylate) matrix ${ }^{16}$. OSSLs with DFB structure can detect nanoscale materials on DFB surface and therefore provide an attractive sensing platform for biological and medical applications ${ }^{17}$. A recent paper discusses the design and performance of organic DFB lasers in biosensor applications ${ }^{17}$.

Organic materials have features that enable the easy fabrication of corrugation structures for DFB and DBR resonators using photochromic materials, photoresists, and photopolymers. The height and width of the corrugation structures can be easily controlled using the illumination interference of the laser beams. The width of the corrugation structures, equivalent to the grating pitch, is related to the emission modes $m=1,2$, or 3 . The height of the corrugation structures, equivalent to the grating depth, is related to the amount of feedback from the lasing light in a micro-cavity, which affects the lasing threshold and slope efficiency.

Although the significance of the grating depth and grating width on the lasing performance of DFB and DBR lasers has been well recognized, only a few studies have discussed these issues ${ }^{18-20}$.

In this report, we fabricated corrugated DFB structures using the same procedure described in our previous reports $^{21,22}$, investigated the lasing characteristics of threshold and slope efficiency as a function of the grating depth, and compared our results with previous results ${ }^{18,19}$.

${ }^{1}$ Faculty of Materials Science and Engineering, Kyoto Institute of Technology, Matsugasaki, Sakyo, Kyoto 606-8585, Japan. ${ }^{2}$ Department of Macromolecular Science and Engineering, Graduate School of Science and Technology, Kyoto Institute of Technology, Matsugasaki, Sakyo, Kyoto 606-8585, Japan. Correspondence and requests for materials should be addressed to N.T. (email: tsutsumi@kit.ac.jp) 


\begin{tabular}{|l|c|c|c|}
\hline Film & RI @ 632.8 nm & RI @ 830 nm & $\boldsymbol{\lambda}_{\mathbf{0}}(\mathbf{n m})$ \\
\hline R6G doped CA & 1.4736 & 1.4712 & 280 \\
\hline SU-8 2002 & 1.587 & 1.578 & 291 \\
\hline ma-P 1275 & 1.6138 & 1.6107 & 344 \\
\hline PVA & 1.5391 & 1.5264 & 200 \\
\hline
\end{tabular}

Table 1. Refractive indices (RI) and $\lambda_{0}$ for R6G doped CA, SU-8 2002, ma-P 1275, and PVA films.

\section{Materials and Methods}

Materials. Two types of photoresists were used for the fabrication of a DFB: a negative type photoresist, SU-8 2002 (MicroChem), and a positive type photoresist, ma-P 1275 (Micro Resist Technology). Photoresist SU-8 2002 consisted of epoxy resin, photo acid generator, and cyclopentanone solvent. Photoresist ma-P 1275 consisted of novolac resin and the photoactive compound diazonaphthoquinone (DNQ). Rhodamine 6G (R6G) (Aldrich Chem.) was used as the laser dye. Cellulose acetate (CA, Aldrich Chem.) was used as the waveguide matrix in the laser device. Diacetone alcohol (DAA, Nacalai Tesque, Japan) was used as the solvent for CA. Polyvinyl alcohol (PVA, Nakalai Tesque, Japan) was used as an interlayer.

Laser device with DFB structure. A solution of SU-8 2002/cyclopentanone (33/67 wt. \%) was spin coated on a quartz substrate at $200-500 \mathrm{rpm}$ for $10 \mathrm{~s}$ followed by $2000 \mathrm{rpm}$ for $12 \mathrm{~s}$. The obtained spin-coated film was pre-baked at $65^{\circ} \mathrm{C}$ for $7 \mathrm{~min}$ and then at $95^{\circ} \mathrm{C}$ for $14 \mathrm{~min}$. An interference beam was illuminated on the SU- 8 thin film using a Lloyd mirror configuration. To adjust the grating pitch, the thin film was set at an appropriate angle. A frequency-tripled Nd:YAG pulse laser delivering a $30 \mathrm{ps}$ pulse at $355 \mathrm{~nm}$ with a $10 \mathrm{~Hz}$ repetition rate was used. After illumination, the SU-8 thin film was post-baked at $65^{\circ} \mathrm{C}$ for $7 \mathrm{~min}$ and then at $95^{\circ} \mathrm{C}$ for $7 \mathrm{~min}$. The SU-8 thin film was developed in an SU-8 Developer for $1 \mathrm{~min}$ in an ultrasonic bath and then rinsed in 2-propanol for $30 \mathrm{~s}$ and dried before heating at $175^{\circ} \mathrm{C}$ for $1 \mathrm{~h}$.

A solution of ma-P $1275 / \mathrm{ma}-\mathrm{T} 1030$ (30/70 wt. \%) was spin coated on a quartz substrate at $500 \mathrm{rpm}$ for $10 \mathrm{~s}$ followed by $3000 \mathrm{rpm}$ for $200 \mathrm{~s}$. To remove the solvent, the ma-P 1275 thin film was heated on a hot plate at $100^{\circ} \mathrm{C}$ for $10 \mathrm{~min}$. An interference beam was illuminated on the ma-P 1275 thin film as described above. The ma-P 1275 thin film was developed in ma-D 331 for $1 \mathrm{~min}$ and rinsed with distilled water for $30 \mathrm{~s}$. The obtained DFB structures were measured using an atomic force microscope (AFM, Nano-R, Pacific Technology).

PVA solution was spin coated on ma-P 1275 DFB to form a $0.2 \mu \mathrm{m}$ PVA interlayer. The PVA interlayer protects the soluble ma-P 1275 DFB structure. A $0.5 \mathrm{wt}$. \% R6G-doped cellulose acetate (CA) solution was spin coated on an SU-8 DFB structure or a PVA-coated ma-P 1275 DFB structure to fabricate an active laser waveguide device. The typical thickness of SU-8 and ma-P 1275 is $0.3 \mu \mathrm{m}$, and that of the active layer is $1.7 \mu \mathrm{m}$.

DFB structures was primarily fabricated using SU-8 photoresist except for the case that ma-P 1275 was used for the grating depth of 15 and $30 \mathrm{~nm}$ for $m=1$. ma-P 1275 photoresist is preferred to fabricate deeper grating depth with wide incidence angle for $m=1$.

Laser emission. The frequency-doubled Nd:YAG pulse laser described above delivering a 30 ps pulse at $532 \mathrm{~nm}$ was used as the pumping laser source. For laser emission measurement, pumping laser beam was focused to a stripe shaped exciting beam with an area of $2.145 \times 10^{-3} \mathrm{~cm}^{2}$ (a length of $33 \times 10^{-4} \mathrm{~cm}$ and a width of $0.65 \mathrm{~cm})$ using a cylindrical lens $(\mathrm{f}=300 \mathrm{~mm})$. The amplified spontaneous emissions (ASE) and lasing emissions from the edge of the waveguide were collected through a quartz optical fibre and monitored using a multi-channel analyser equipped with an Andor iDus charge coupled device (CCD) and gratings (1200 lines/500 nm). Pumping laser energy reflected by a half-mirror was monitored an Ophir photodiode type pyrometer PD10 with Nova II Display. Lasing beam energy emitted from the edge of the waveguide was monitored by an optical fibre coupled photodiode pyrometer (Ophir PD10-PJ with Nova II Display) equipped with an optical filter to cut an exciting beam in front ${ }^{23}$.

Characterization. The absorption spectrum of a sample film was measured using a UV and visible spectrometer (UV-2100PC, Shimadzu, Japan). The photoemission spectrum of a sample film was recorded using a Shimadzu RF-1500 fluorophotometer. The film thickness was measured using an AFM.

The refractive indices of R6G doped CA, PVA, and ma-P 1275 films was measured at $632.8 \mathrm{~nm}$ and $830 \mathrm{~nm}$ using an $\mathrm{m}$-line technique of the prism coupling method. A prism and waveguide were coupled with an air-gap. A He-Ne laser at $632.8 \mathrm{~nm}$ and a laser diode at $830 \mathrm{~nm}$ were used as laser sources for the prism coupling method. Refractive indices of R6G doped CA, SU-8 2002, PVA, and ma-P 1275 films were measured in TE mode at 632.8 and $830 \mathrm{~nm}$ are shown in Table 1 . Index of refraction at some wavelength $n(\lambda)$ was evaluated using a one-oscillator Sellmeier-dispersion formula as follows:

$$
n(\lambda)^{2}-1=\frac{q}{1 / \lambda_{0}^{2}-1 / \lambda^{2}}+A
$$

where $q$ is a measure of the oscillator strength, $A$ is a constant containing the sum of all other oscillators, and $\lambda_{0}$ is a absorption wavelength of dominant oscillator. The value of $\lambda_{0}$ for each film is shown in Table 1 . The values of $q$ and $A$ were calculated for each film with the values of refractive index and $\lambda_{0}$ listed in Table 1 . Refractive index of lasing wavelength region around $590 \mathrm{~nm}, n=1.48$ for R6G doped CA, $n=1.59$ for SU-8 2002, $n=1.62$ for ma-P 1275 , and $n=1.54$ for PVA were evaluated. 
4 layer (Air/Active/SU-8/Substrate)

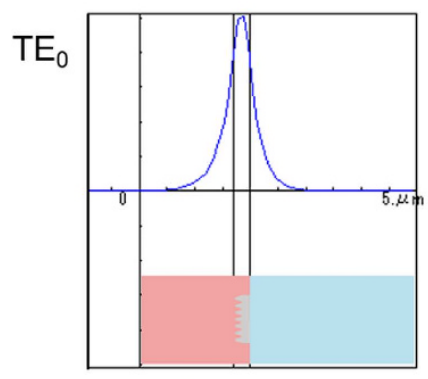

$\mathrm{TE}_{1}$

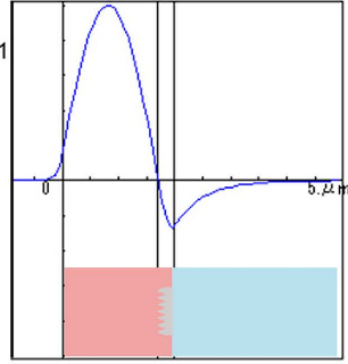

5 layer (Air/Active/PVA/ma-P/Substrate)
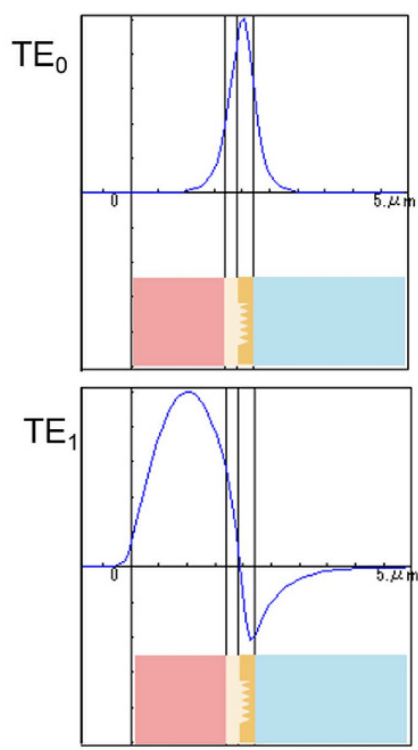

Figure 1. Optical confinement (blue curve) in four-layer and five-layer waveguides. Optical confinement was calculated using an NL-guide waveguide simulation software. For both waveguides, light is confined in photoresists with higher refractive indices for the $\mathrm{TE}_{0}$ mode, whereas light is confined in the active layer and gain medium for the $\mathrm{TE}_{1}$ mode.

\section{Results and Discussion}

Waveguide modes. The simplest waveguide laser is a three-layer structure that consists of: air, a core with a gain medium and a clad layer with relief grating structures. In the presented waveguide laser device with DFB structures, four- and five-layer waveguides were employed. The four-layer waveguide consisted of air $(n=1) / \mathrm{R} 6 \mathrm{G}$ doped CA gain medium $(n=1.48) / \mathrm{SU}-82002(n=1.59)$ with a grating structure/Quartz substrate $(n=1.46)$. The five-layer waveguide consisted of air $(n=1) / \mathrm{R} 6 \mathrm{G}$ doped CA gain medium $(n=1.48) / \mathrm{PVA}$ intermediate layer $(n=1.54) /$ ma-p $1275(n=1.62)$ with a gain medium/Quartz substrate $(n=1.46)$. The refractive index of the waveguide core, R6G doped CA, is 1.48, which is lower than those of the SU-8 $2002(n=1.59)$, ma-P $1275(n=1.62)$, and PVA interlayer $(n=1.54)$. The confinement of light is calculated using a waveguide simulated by an NL-guide software. The confinement of light in the waveguide is shown in Fig. 1. In the $\mathrm{TE}_{0}$ waveguide mode, light is confined in the SU-8 2002 layer because of the high refractive index of SU-8 2002, 1.59. Whereas, in the $\mathrm{TE}_{1}$ waveguide mode, light is confined in the CA active layer with the R6G laser dye possessing a refractive index of 1.48.

The same type of situation is evaluated in a five-layer waveguide. In a similar manner, light is confined in PVA and ma-P 1275 in the $\mathrm{TE}_{0}$ waveguide mode, whereas it is confined in the active layer in the $\mathrm{TE}_{1}$ mode. The thicknesses of the PVA and ma-P 1275 layers are 0.2 and $0.3 \mu \mathrm{m}$, respectively.

Thus, the preferential waveguide mode for the present $\mathrm{DFB}$ laser is determined to be the $\mathrm{TE}_{1}$ mode for both photoresist waveguides.

Fabrication of grating structures. In DFB lasers, the properties of the grating structures (grating depth and grating pitch) are important for lasing performance. The grating pitch is directly related to the mode number for diffraction. Figure 2 shows the absorption, fluorescence, and amplified spontaneous emission (ASE) spectra of $0.5 \mathrm{wt} \%$ R6G in the CA film. The thickness of the film was $1.49 \mu \mathrm{m}$, which was determined using a Lambert-Beer law. Extinction coefficient of R6G is $116,000 \mathrm{~cm}^{-1} \mathrm{M}^{-1}$ at peak wavelength ${ }^{24}$. Density of CA is $1.3 \mathrm{~kg} \mathrm{dm}^{-3}$. Molecular weight of R6G is $479.02 \mathrm{~g} \mathrm{~mol}^{-1}$. Absorbance of spin-coated film was 0.234 at peak wavelength. The ASE ranges from 570 to $610 \mathrm{~nm}$. Lasing occurs in the ASE range. Thus the grating pitch equivalent to the resonance length of the micro-cavity in the DFB structures should be determined. The grating pitch, which is the same as the resonance length of the micro-cavity $L$ in DFB structures is calculated as follows:

$$
L=\frac{m}{2} \times \frac{\lambda_{\mathrm{L}}}{n_{\mathrm{eff}}}
$$

where $m$ is the diffraction mode, $\lambda_{\mathrm{L}}$ is the lasing wavelength, and $n_{\text {eff }}$ is the effective refractive index. Typically, the grating pitch is $200 \mathrm{~nm}$ for $m=1,400 \mathrm{~nm}$ for $m=2$, and $600 \mathrm{~nm}$ for $m=3$, with $\lambda_{\mathrm{L}}=600 \mathrm{~nm}$ and $n_{\text {eff }}=1.5$. There are several ways to fabricate the grating in photoresists: electron lithography, direct laser writing, and by the interference of laser beams. In this report, the interference of two beams method was employed. To fabricate grating pitches of 200,400 , and $600 \mathrm{~nm}$, the incidence angles of the interference beam were $62.6^{\circ}, 26.3^{\circ}$, and $17.2^{\circ}$, respectively. Figure 3 shows AFM images of fabricated gratings in SU-8 photoresist for the mode numbers (a) $m=1$, (b) $m=2$, and (c) $m=3$. The grating depth profile for each mode is also shown in Fig. 3. The grating depth becomes shallower with wider incidence angles. 

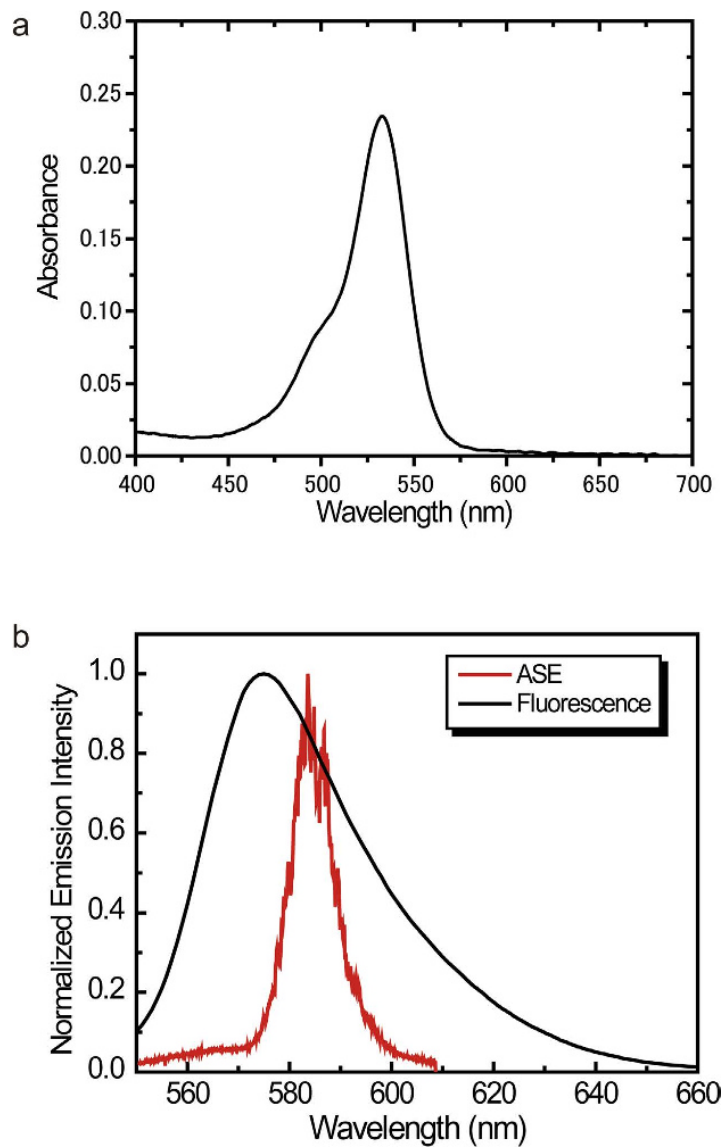

Figure 2. Absorption, photoluminescence, and ASE spectra of Rhodamine 6G in a CA thin film. (a) Absorption spectrum. (b) Fluorescence and ASE spectra. Fluorescence broadens from 550 to over $650 \mathrm{~nm}$. ASE is in the range from 570 to $610 \mathrm{~nm}$.

Lasing threshold and slope efficiency. A typical DFB laser device is shown in Fig. 4(a). The corrugated structure was fabricated in SU-8 photoresist and it was located $6.5 \mathrm{~mm}$ inside the substrate edge using the Lloyd mirror technique.

The threshold and slope efficiency of DFB lasing are measured to evaluate the lasing performance of the DFB lasers. The lasing threshold was determined by the pump energy at which a narrow lasing peak appeared. Figure 4(b) is a typical lasing peak profile for DFB lasing when the pump energy is changed from 26 to $65 \mu \mathrm{J} \mathrm{cm}{ }^{-2}$ pulse $\mathrm{e}^{-1}$ for the mode $m=1$. The lasing wavelength is $587.4 \mathrm{~nm}$ and the lasing width is $<0.2 \mathrm{~nm}$. The emission intensity is plotted as a function of the pump energy in Fig. 4(c). The lasing intensity increases linearly with increasing pump energy. The lasing threshold is determined by the intersection of two lines, as shown in Fig. 4(c). A lasing threshold of $27 \mu \mathrm{J} \mathrm{cm}^{-2}$ pulse $\mathrm{c}^{-1}(58 \mathrm{~nJ})$ is evaluated from the intersection of two lines. Figure 5 shows the dependence of the lasing threshold on the grating depth for the modes $m=1, m=2$, and $m=3$. All corrugated structures were fabricated by SU-8 photoresist. The lasing threshold for $m=1$ is lower than that for $m=2$ and $m=3$. This result is reasonable because significant emission loss from the waveguide due to the radiation loss (radiation mode) should be considered for higher modes $m \geq 2$.

The slope efficiency is determined by the slope between the pump (input) energy and output energy. The output energy is plotted as a function of the input energy for the mode $m=1$ with a grating height of $15 \mathrm{~nm}$ and a grating pitch of $189 \mathrm{~nm}$, as shown in Fig. 6. The slopes of the plots give the slope efficiency, which is $1.6 \%$ in this case.

For feedback lasing using DFB structures, the grating depth (height) is important to determine the feedback of light in the feedback cavity. Thus the slope efficiency is investigated as a function of the grating depth (height). The grating depth was varied with the change in the period of illumination. We can vary the grating depth (height) between 5 and $30 \mathrm{~nm}$ for the mode $m=1$ using an interference technique with a grating pitch between 189 and $200 \mathrm{~nm}$. Su- 8 photoresist was used except for the case that ma-P 1275 photoresist was used for the grating depth of 15 and $30 \mu \mathrm{m}$ for $m=1$. A slope efficiency of $0.33 \%$ for the mode $m=2$ was measured for the sample with a grating pitch of $391 \mathrm{~nm}$ and a grating depth of $8.5 \mathrm{~nm}$. A slope efficiency of $0.43 \%$ for the mode $m=3$ was measured for the sample with a grating pitch of $590 \mathrm{~nm}$ and a grating depth of $55 \mathrm{~nm}$. The slope efficiencies are plotted together as a function of the grating depth in Fig. 7. Several recent papers have also discussed the relationship between the slope efficiency and the grating depth ${ }^{18,19}$ in DFB lasing. Recent results published by other researchers ${ }^{18}$ are plotted in Fig. 7 as a reference. The laser active material was the polyfluorene derivative poly[(9,9-dioctylfluorenyl-2,7-diyl)-co-(1,4-benzo\{2,10,3\}-thiadiazol)] (F8BT) doped with 
(a)
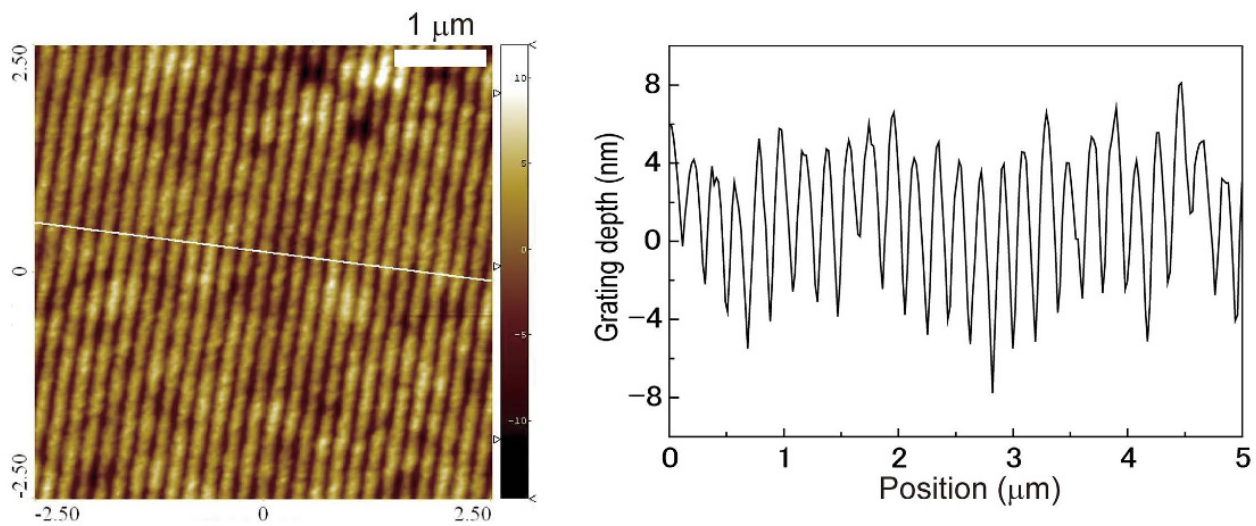

(b)
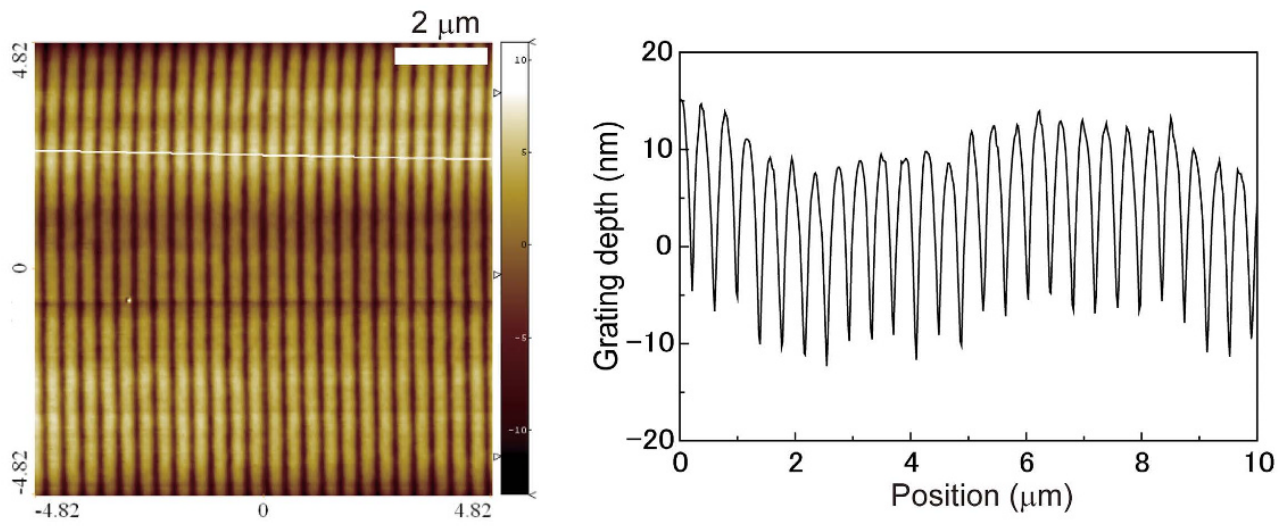

(c)
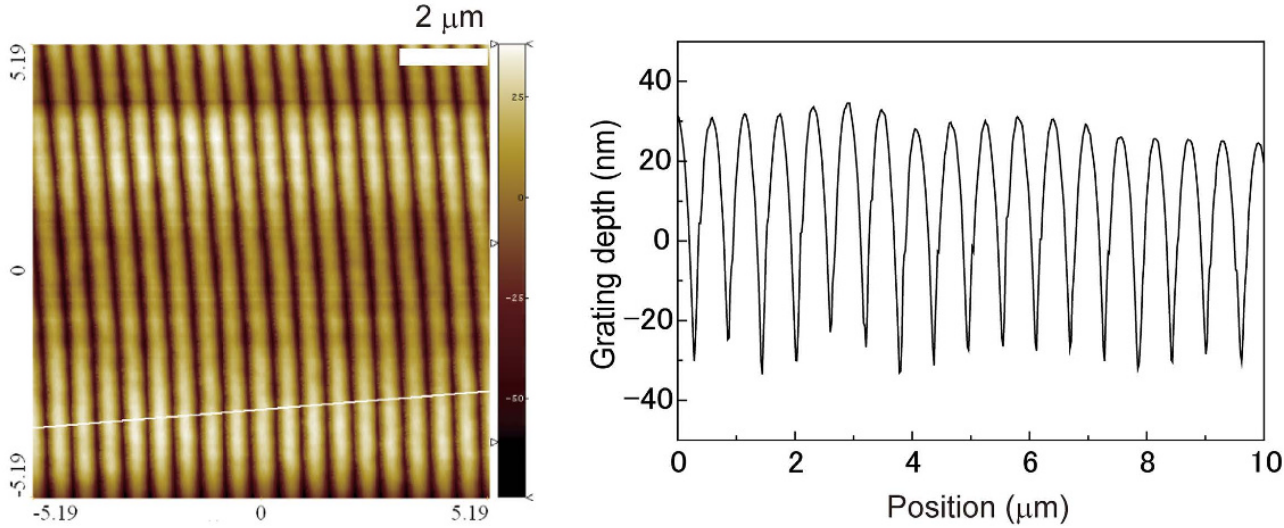

Figure 3. AFM images and grating depths of corrugation structures fabricated in SU-8 photoresist by interference beams. Grating depth is measured along a white line in each AFM image. (a) A grating pitch of $199 \mathrm{~nm}$ which corresponds to lasing mode $m=1,(\mathbf{b})$ a grating pitch of $390 \mathrm{~nm}$ which corresponds to lasing mode $m=2$, and (c) a grating pitch of $590 \mathrm{~nm}$ which corresponds to lasing mode $m=3$.

15 wt. \% of poly[2-methoxy-5-(20-ethyl-hexyloxy)-1,4-phenylene vinylene] (MEH-PPV). The grating pitch was $410 \mathrm{~nm}^{18}$, so the lasing mode was assumed to be $m=2$. A lasing slope efficiency of less than $1 \%$ was reported in an R6G-doped SU-8 photoresist in which the grating structure with a grating depth of $80 \mathrm{~nm}$ and a grating pitch of $550 \mathrm{~nm}$ (equivalent to the mode $m=3$ ) was fabricated by two-photon laser fabrication ${ }^{19}$. These results are in good agreement with the present results for the mode $m=3$.

The coupling of waves is defined by the coupling coefficient $\kappa$ as follows:

$$
\kappa=\frac{\pi h\left(n_{\mathrm{f}}^{2}-n_{\mathrm{eff}}^{2}\right)}{\lambda h_{\mathrm{eff}} \lambda_{\mathrm{eff}}}
$$


a
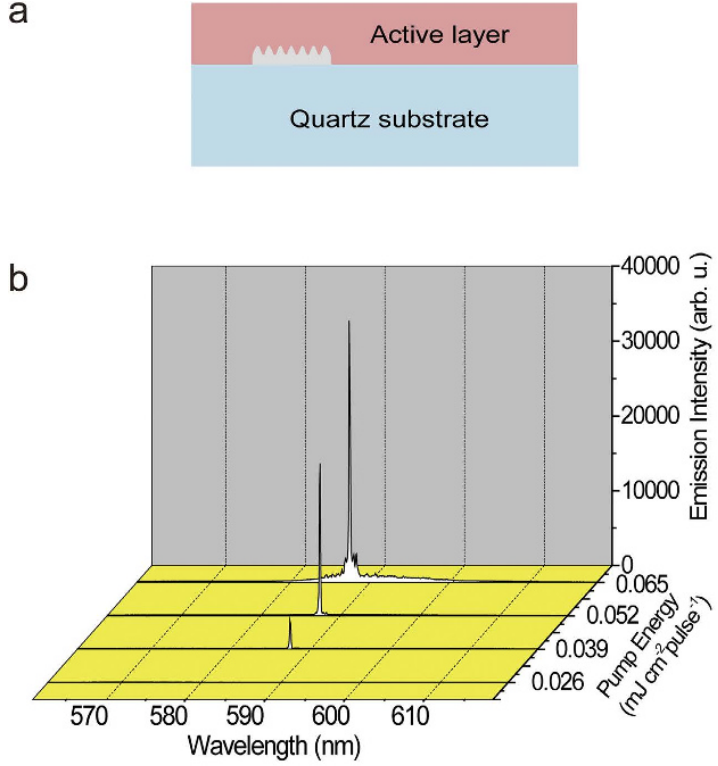

C

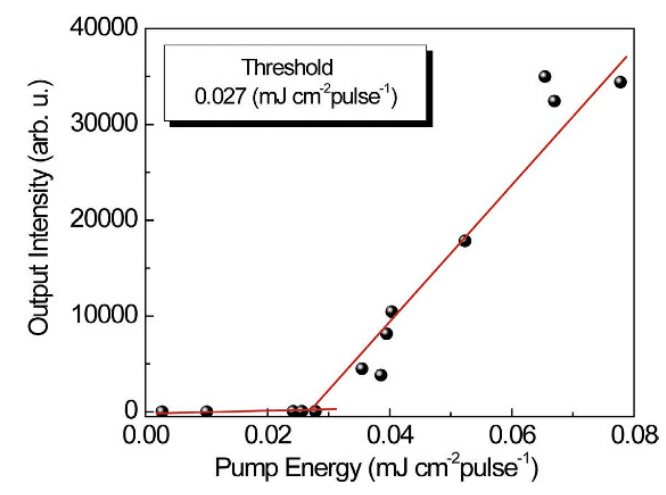

Figure 4. (a) Schematic diagram of DFB laser device. Corrugation structures of SU-8 photoresist were fabricated $6.5 \mathrm{~mm}$ inside the edge. (b) Lasing peak profile of DFB laser with mode $m=1$. Emission wavelength is $587.4 \mathrm{~nm}$. Lasing occurred at $587.4 \mathrm{~nm}$ with the DFB structures fabricated using the incidence angle of $65.5^{\circ}$. (c) Plots of emission intensity as a function of pump energy. Intersect of the two lines gives the lasing threshold.

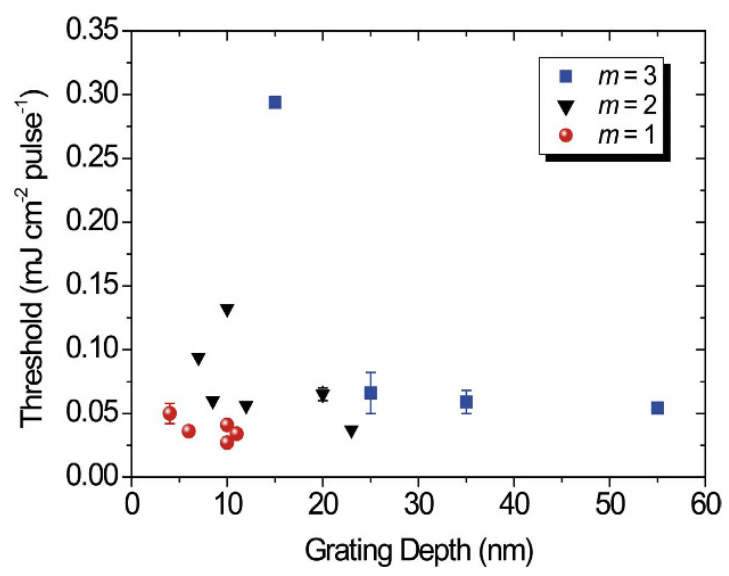

Figure 5. Plots of lasing threshold as a function of grating depth for $m=1$ (red circle plots), $m=2$ (black inverse triangle plots), and $\boldsymbol{m}=\mathbf{3}$ (blue square plot). A lower mode number gives a lower threshold. All corrugated structures are fabricated by SU-8 photoresist. 


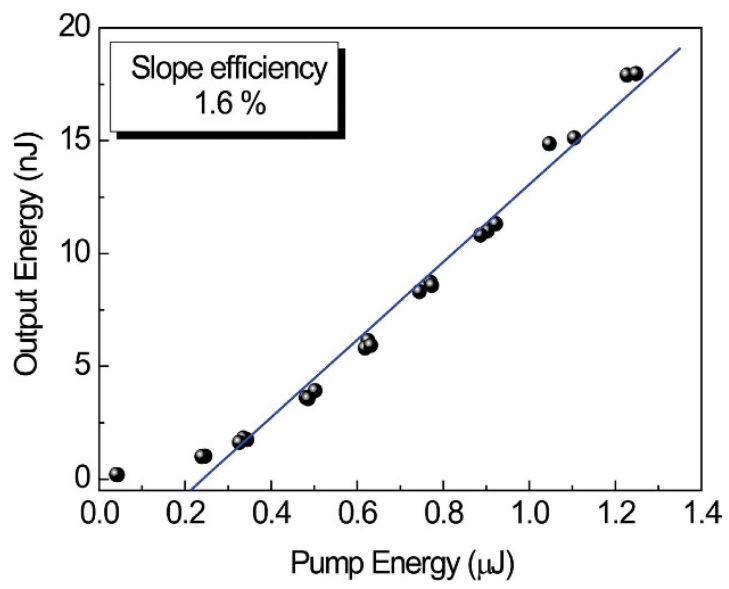

Figure 6. Plots of pump energy and emitted (output) energy. Blue line is a slope efficiency of $1.6 \%$. Corrugated structure is fabricated in SU-8 photoresist.

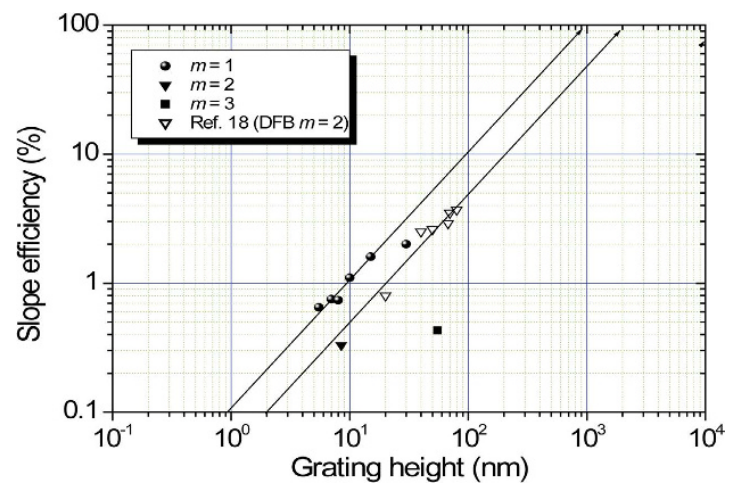

Figure 7. The plots of slope efficiency as a function of grating depth. The black circle is the slope efficiency for lasing mode $m=1$, the black inverse triangle is for $m=2$, and the black square is for $m=3$. The white inverse triangle is taken from ref. 18 and replotted. Gain matrix is F8BT doped by MEH-PPV.

$$
h_{\mathrm{eff}} \approx \frac{\lambda}{\sqrt{n_{\mathrm{f}}^{2}-n_{\mathrm{s}}^{2}}},
$$

where $h$ is equivalent to the grating height, $h_{\text {eff }}$ is the minimum effective film thickness of the fundamental mode, $n_{\mathrm{f}}$ is the refractive index of the active layer and $n_{\mathrm{s}}$ is the refractive index of the substrate ${ }^{18}$.

The feedback gain $G$ is the product of the coupling coefficient and the resonance length of the micro-cavity $L^{25-27} . L$ increases linearly with the diffraction mode for lasing $m$.

$$
G=\kappa L \propto h m
$$

Thus, larger values of $h$ and deeper grating depths result in larger feedback gains, which lead to efficient lasing performance and a higher slope efficiency. The straight line in Fig. 7 gives the linear relation between the slope efficiency and grating depth. If the feedback gain is parallel to the slope efficiency, a grating depth of $100 \mathrm{~nm}$ achieves a slope efficiency of $10 \%$ and a $1000 \mathrm{~nm}$ grating depth achieves a $100 \%$ slope efficiency for the mode $m=1$. Direct laser writing is promising for the fabrication of high aspect ratio grating structures to achieve a high lasing slope efficiency.

We must also consider the contradictory relationships: a higher diffraction mode leads to a larger optical loss due to the radiation loss of the feedback light from the waveguide (radiation mode). In other words, for the diffraction mode higher than $m=2$, part of the feedback light is radiated from the waveguide as a radiation mode, which diminishes the lasing performance. This situation can be predicted from Fig. 7 .

\section{Conclusions and Outlook}

We investigated the lasing characteristics, i.e., threshold and slope efficiency, as a function of the grating depth in OSSL devices with a DFB resonator fabricated using photoresists. The threshold for the first order mode $(m=1)$ was lower than that for the second and third order modes $(m=2$, and 3$)$. A low threshold of $27 \mu \mathrm{J} \mathrm{cm}^{-2}$ pulse $^{-1}$ $(58 \mathrm{~nJ})$ was obtained using SU-8 2002 with the mode $m=1$. The slope efficiency was evaluated as a function of the 
grating depth for each mode. The slope efficiency increased with increasing grating depth, which can be explained by the increase in the coupling gain. In the higher modes greater than 2 , the radiation loss reduced the lasing performances. Thus the mode number $m=1$ gives the highest lasing performance (lowest threshold and highest slope efficiency). The present report assumes that a grating depth of $100 \mathrm{~nm}$ achieves a $10 \%$ slope efficiency and a grating depth of $1000 \mathrm{~nm}$ achieves a $100 \%$ slope efficiency for the mode $m=1$.

\section{References}

1. Samuel, I. D. W. Laser physics: Fantastic plastic. Nature 429(6993), 709-711 (2004).

2. Samuel, I. D. W. \& Turnbull G. A. Organic semiconductor lasers. Chem. Rev. 107, 1272-1295 (2007).

3. Chénais, S. \& Forget, S. Recent advances in solid-state organic lasers. Polym. Int. 61, 390-406 (2012).

4. Coles, H. \& Morris, S. Liquid-crystal lasers. Nat. Photonics 4, 676-685 (2010).

5. Tsutsumi, N. \& Shinobu, M. All organic DFB laser enhanced by intermediate high refractive index polymer layer. Appl. Phys. B 105, 839-845 (2011).

6. Mhibik, Q., Leang, T., Siove, A., Forget, S. \& Chénais, S. Broadly tunable (440-670 nm) solid-state organic laser with disposable capsules. Appl. Phys. Lett. 102, 041112 (2013).

7. Huang, W., Chen, L. \& Xuan, L. Efficient laser emission from organic semiconductor activated holographic polymer dispersed liquid crystal transmission gratings. RSC Adv. 4, 38606-38613 (2014).

8. Diao, Z. et al. A dual-wavelength surface-emitting distributed feedback laser from a holographic grating with an organic semiconducting gain and a doped dye. J. Mater. Chem. C 2, 6177-6182 (2014).

9. Huang, W., Liu, Q., Xuan, L. \& Chen, L. Single-mode lasing from dye-doped holographic polymer-dispersed liquid crystal transmission gratings. Appl. Phys. B 117, 1065-1071 (2014).

10. Kim, J.-H. et al. Tunable and flexible solvent-free liquid organic distributed feedback lasers. Appl. Phys. Lett. 106, 053302 (2015).

11. Yoshioka, H. et al. Extreme ultra-low lasing threshold of full-polymeric fundamental microdisk printed with room-temperature atmospheric ink-jet technique. Scientific Reports 5, 10623 (2015).

12. Boj, P. G. et al. Organic distributed feedback laser to monitor solvent extraction upon thermal annealing in solution-processed polymer films. Sensors and Actuators B: Chemical 232, 605-610 (2016).

13. Smirnova, T. N., Sakhno, O. V., Stumpe, J. \& Fitio, V. M. Polymer distributed feedback dye laser with an external volume Bragg grating inscribed in a nanocomposite by holographic technique. J. Opt. Soc. Am. B 33(2), 202-210 (2016).

14. Zhao, Z., Mhibik, O., Nafa, M., Chénais, S. \& Forget, S. High brightness diode-pumped organic solid-state laser. Appl. Phys. Lett. 106, $051112(2015)$.

15. Mhibik, O. et al. An ultra-narrow linewidth solution-processed organic laser. Light Sci. Appl. 5, e16026 (2016).

16. Yoshioka, H., Yang, Y., Watanabe, H. \& Oki, Y. Fundamental characteristics of degradation recoverable solid-state DFB polymer laser. Opt. Express 20(4), 4690-4696 (2012).

17. Haughey, A. M. et al. Organic semiconductor laser biosensor: Design and performance discussion. IEEE J Selected Topics Quantum Electronics 22, 1300109 (2016).

18. Döring, S. Rabe, T. \& Stumpe, J. Output characteristics of organic distributed feedback lasers with varying grating heights. Appl. Phys. Lett. 104, 263302 (2014).

19. Horn, W., Kroesen, S. \& Denz, C. Two-photon fabrication of organic solid-state distributed feedback lasers in rhodamine 6G doped SU-8. Appl. Phys. B 117, 311-315 (2014).

20. Navarro-Fuster, V. et al. Film thickness and grating depth variation in organic second-order distributed feedback lasers. J. Appl. Phys. 112, 043104 (2012).

21. Tsutsumi, N. \& Takeuchi, M. Ti-sapphire femtosecond pulse pumped laser emission from all-plastic organic waveguide with distributed feedback resonator. Optics Communications 281, 2179-2183 (2008).

22. Tsutsumi, N., Takeuchi, M. \& Sakai, W. All-plastic organic dye laser with distributed feedback resonator structure. Thin Solid Films 516, 2783-2787 (2008)

23. Tsutsumi, N. \& Shinobu, M. All organic DFB laser enhanced by intermediate high refractive index polymer layer. Appl. Phys. B 105, 839-845 (2011).

24. Birge, R. R. Kodak Laser Dyes. Kodak publication JJ-169 (1987)

25. David, K.et al. Gain-coupled DFB lasers versus index-coupled and phase-shifted DFB lasers: a comparison based on spatial hole burning corrected yield. IEEE J Quantum Electronics 27(6), 1714-1723 (1991).

26. Lowery, A. J. \& Novak, D. Performance comparison of gain-coupled and index-coupled DFB semiconductor lasers. IEEE J Quantum Electronics 30(9), 2051-2063 (1994).

27. Ebeling, K. J. Integrated Optoelectronics Springer, Berlin, Heidelberg, New York (1996).

\section{Author Contributions}

N.T. contributed to designing the study and setting up the optical measurements. S.N. contributed to preparing the sample device and performing the optical measurements. N.T. contributed to preparing the manuscript. K.K and W.S. contributed to fruitful discussions.

\section{Additional Information}

Competing financial interests: The authors declare no competing financial interests.

How to cite this article: Tsutsumi, N. et al. Re-evaluation of all-plastic organic dye laser with DFB structure fabricated using photoresists. Sci. Rep. 6, 34741; doi: 10.1038/srep34741 (2016).

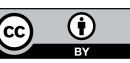

This work is licensed under a Creative Commons Attribution 4.0 International License. The images or other third party material in this article are included in the article's Creative Commons license, unless indicated otherwise in the credit line; if the material is not included under the Creative Commons license, users will need to obtain permission from the license holder to reproduce the material. To view a copy of this license, visit http://creativecommons.org/licenses/by/4.0/

(C) The Author(s) 2016 